\title{
Inflammatory Myofibroblastic Tumour of Ovary Simulating Malignancy with Review of Literature
}

\author{
Ruchi Sinha, Shuchismita, Iffat Jamal*, Shashikant Kumar, Nishi and Mamta Kumari \\ Department Of Pathology, All India Institute Of Medical Sciences-Patna(India)
}

\begin{abstract}
Inflammatory myofibroblastic tumour (IMT) of ovary is an uncommon benign neoplasm that can mimic malignancy. It often occurs in children and young adults. Lung is the most common site of IMT. Precise etiology is not known but recent reports suggested the role of benign reactive process in its pathogenesis.

A 32 year old female patient presented to the gynaecology out patient department with complaints of lower abdominal pain and increased frequency of micturition with burning since 15 days. On per abdominal examination, an irregular lump of 18 weeks size with restricted mobility was palpable in suprapubic region. Laboratoratory investigations revealed normocytic normochromic anaemia with neutophilic leucocytosis and raised ESR. Her serum CA 125 levels were slightly raised $(44.30 \mathrm{u} / \mathrm{ml})$. Imaging studies showed an infiltrating mass in the pelvis. The patient underwent total abdominal hysterectomy with bilateral salpingo oopherectomy and omentectomy. Histopathological examination of which revealed IMT of right ovary with extension to right parametrium and adjacent bladder.

As IMT is a mimicker of malignancy due to its infiltrative growth. Histopathological examination is mandatory for its diagnosis and treatment. Misdiagnosis can lead to unnecessary extensive debulking surgeries and even chemotherapy. Rarity of this entity in the ovary has made this case worth reporting.
\end{abstract}

Keywords: Inflammatory, Myofibroblastic, Tumour, Malignancy, Salpingo-Oopherecromy

\section{Introduction}

Inflammatory myofibroblastic tumour (IMT), also known as plasma cell granuloma, plasma cell pseudo tumour, inflammatory myofibrohistiocytic proliferation and inflammatory pseudotumour is a rare benign lesion of mesenchymal origin. The designation of IMT was adopted by the World Health Organization (WHO) classification of tumours of the soft tissue and bone in the year 2013. ${ }^{[1]}$ It primarily affects children and young adults. ${ }^{[1]}$ Precise etiology of this lesion is unknown. The neoplasm is composed of proliferating myofibroblastic spindle cells admixed with chronic inflammatory cells such as lymphocytes, plasma cells and eosinophils. It can arise in any organ but most frequent reported sites are lung, mesentry and omentum. ${ }^{[1]}$ Female genital tract is one of the rarest site for occurance of IMT with most cases reported in the uterus. ${ }^{[2]}$ Only few cases of IMT in ovary have been reported. ${ }^{[3]}$

\section{Case Report}

A 32 year old female presented to the gynaecology out patient department with complaints of lower abdominal pain, burning micturition and increased frequency of micturition since 15 days. There was no history of fever or vomiting. On physical examination of abdomen, a firm irregular lump of 18 weeks size with restricted mobility was palpable. Laboratory investigations revealed low haemoglobin (8.4 gm\%), neutrophilic leukocytosis, raised ESR and slightly raised serum CA 125 level (44.30 U/ml). Culture and sensitivity test of urine did not show any growth. Per vaginal examination revealed a firm lump of restricted mobility measuring $10 \times 10 \mathrm{~cm}$ which appeared as an irregular mass separate from uterus. The rectal mucosa was free on per rectal examination. Peritoneal tap aspirated $40 \mathrm{ml}$ haemorrhagic fluid which on cytological analysis showed inflammatory smear comprising of neutrophils, lymphocytes with few clusters of benign mesothelial cells. No evidence of malignancy was found. Cervical smear was reported as inflammatory cervical smear with bacterial vaginosis. Ultrasonography of abdomen showed a solid cystic pelvic mass measuring $11 \times 9.45 \mathrm{~cm}$. The mass was present anterior to the uterus. Endometrial thickness was $8 \mathrm{~mm}$.

Contrast- enchanced computed tomography (CECT) of the whole abdomen was performed which revealed a large lobulated right ovarian mass $(11 \times 7.5 \times 7.5 \mathrm{~cm})$ involving and adhering to the right anterior uterine wall, extending anteriorly upto left uterine cornu and involving both sides of uterus (FIG -1$)$. The mass was isodense to 
the uterus with similar enhancement pattern. The mass was solid with cystic component. The cyst wall was $1 \mathrm{~cm}$ thick and contained pus/necrotic material. The left ovary showed a $4.5 \times 3.2 \times 2.1 \mathrm{~cm}$ heterogenous mass . The fat and omentum anterior to the right ovarian lump seems to be infiltrated. Few satellite nodules measuring upto $1 \mathrm{~cm}$ in size were seen. Surrounding pelvic viscera appeared to be free on CECT. Enlarged lymph nodes were seen in both iliac chains, largest measuring $20 \times 12 \mathrm{~cm}$ in size. Few enlarged paraotic lymph nodes were present, the largest one measuring $1.5 \times 0.9 \mathrm{~cm}$.

A clinical diagnosis of stage IV-A ovarian carcinoma was given and total abdominal hysterectomy with bilateral salpingo oopherectomy and omentectomy (suboptimal debulking) was planned.

Intra operatively, a solid-cystic mass measuring 5 x $5 \mathrm{~cm}$ was seen in right ovary and filled with approximately 30 $40 \mathrm{ml}$ of pus. The pus was aspirated and sent for culture and sensitivity. The ovarian mass was densely adherent and infiltrating into uterus, bladder, vagina, gut, omentum and extending upto the lateral pelvic wall. Due to the dense adhesions urinary bladder was perforated while removing the ovarian mass and which was subsequently repaired.

Pus culture showed growth of Staphylococcus aureus. Cytology revealed degenerated as well as intact polymorphs admixed with few mononuclear cells comprising of plasma cells and macrophages.

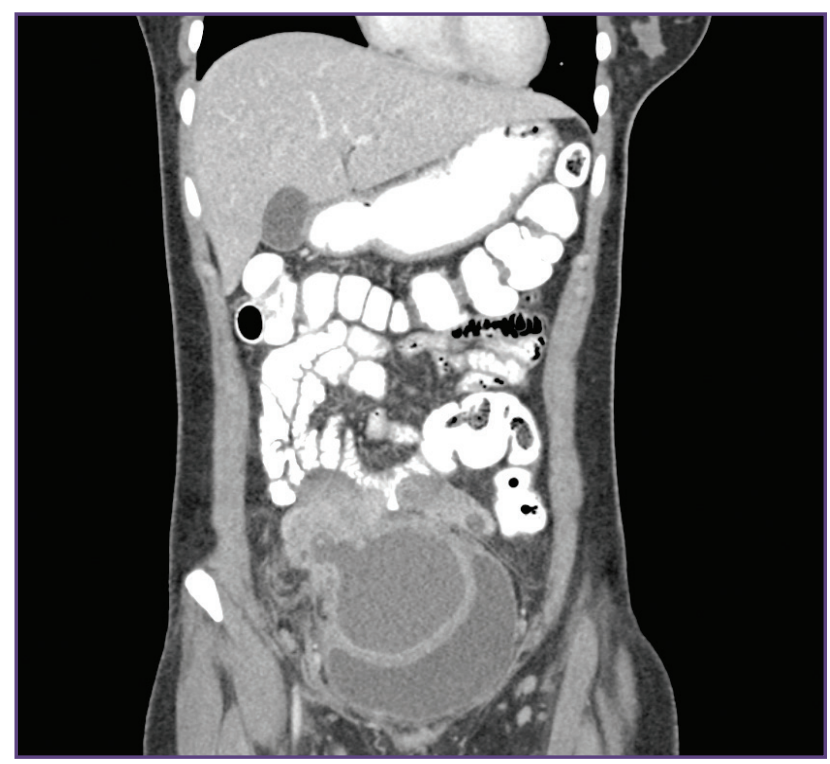

Fig. 1: Computed tomography scan showing a solid cystic right ovarian mass involving and adhering to the right anterior uterine wall, extending anteriorly upto left uterine cornu and involving both sides of uterus.
The excised specimen was sent for histopathological examination. On gross examination, cut section of the mass revealed solid grey white areas with pale yellow patches and cystic spaces (Figure 2). Microscopic examination showed proliferating myofibroblasts (at places arranged in fascicles) along with diffuse infiltration of chronic inflammatory cells comprising of lymphocytes with focal lymphoid aggregates, plasma cells, histiocytes and occasional eosinophils (Figure 3). Focal aggregates of foamy macrophages were seen. Areas of coagulative necrosis as well as sclerosed and congested blood vessels were present. No pleomorphism, atypical mitosis and lymphovascular invasion was noted. The mass was seen to entrap the fat spaces and infiltrating into the muscularis propria of bladder (detrusor muscle). Left ovary was not involved microscopically. Diagnosis of IMT of right ovary with extension to right parametrium, anterior uterine wall, peritoneal fat and detrusor muscle of bladder was given.

Immunohistochemistry showed diffuse positivity for vimentin(FIG 4), smooth muscle actin(Fig.4) was positive in the fibrous septa and blood vessels. ALK was negative. Post operative period was unremarkable.

\section{Discussion}

IMT is a rare benign mesenchymal neoplasm. Proper detailed description of its pathological identity was done in 1954 by Iverson and Umiker, though it was described first by Brunn. ${ }^{[4]}$ It often presents in first and second decades of life with slight female predominance. ${ }^{[1]}$ Lungs are most

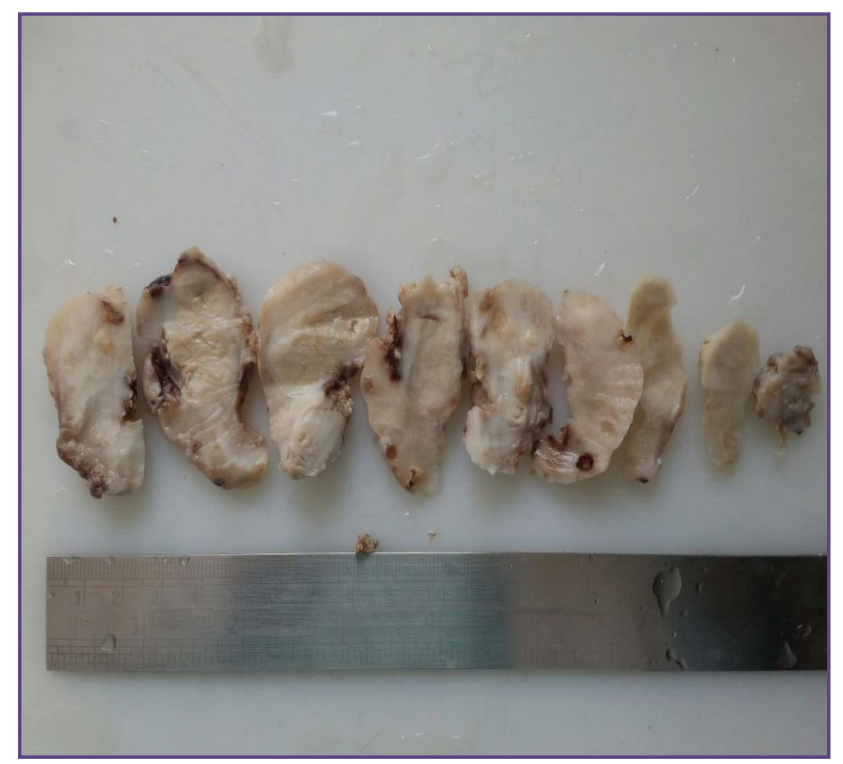

Fig. 2: serial sectioning of the right ovary revealed solid grey white areas with pale yellow patches and cystic spaces. 


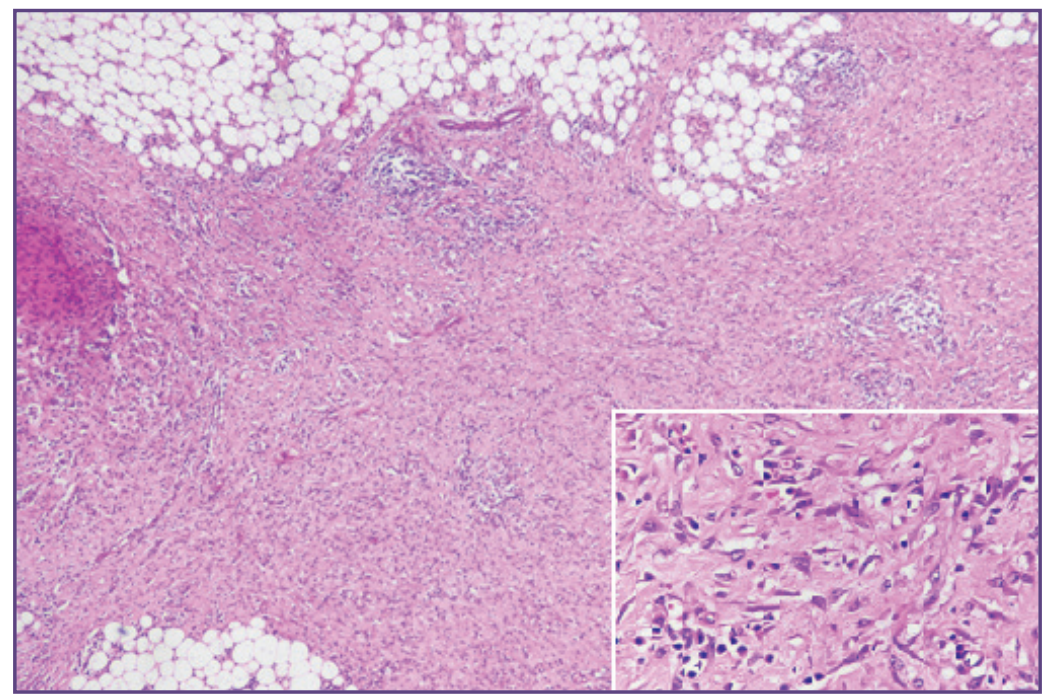

Fig. 3: photomicrograph showing proliferating myofibroblasts along with diffuse infiltrate of chronic inflammatory infiltrate entrapping the fat spaces(H\&E - 10X4). Inset showing the same in higher magnification (H\&E - 10X40).

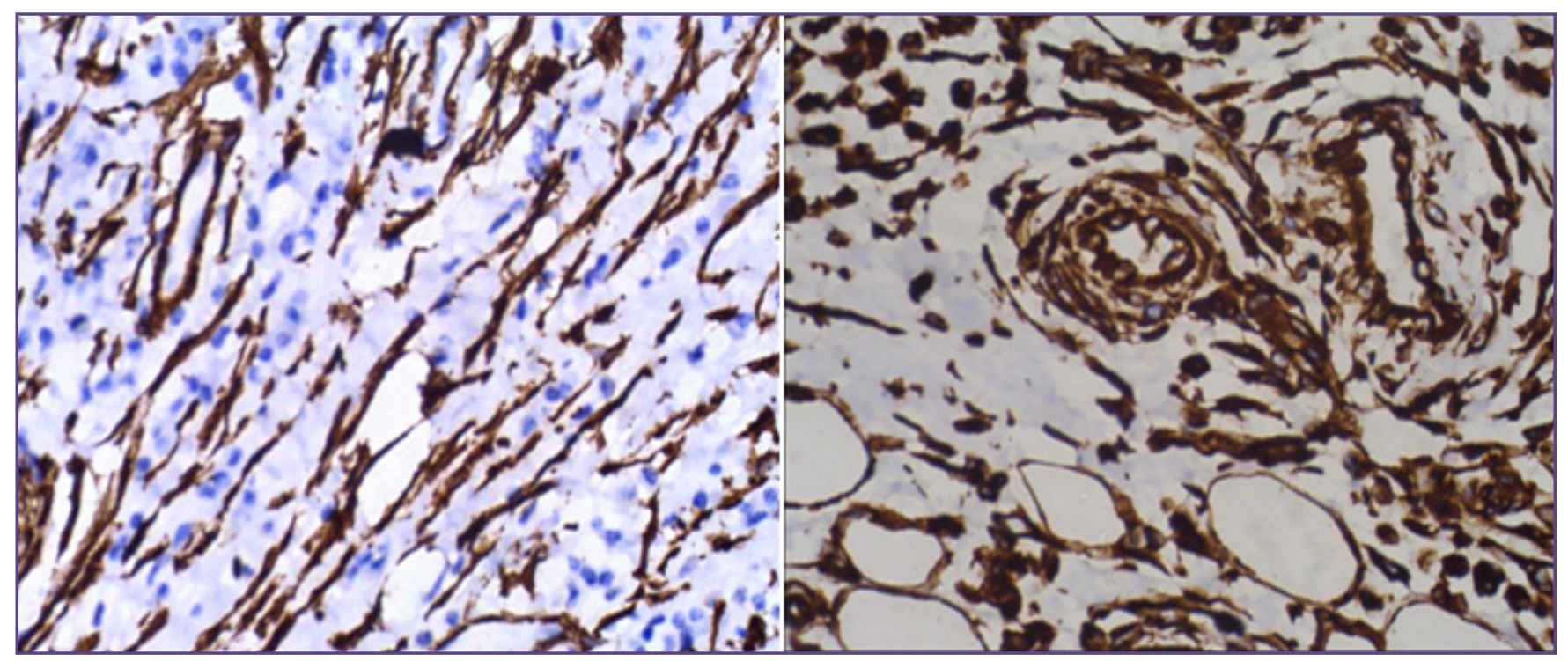

Fig. 4: photomicrograph showing diffuse positivity for smooth muscle actin and vimentin respectively.

frequently involved site. ${ }^{[5]}$ IMTs of female genital tract are rare with uterus being the most common organ. ${ }^{[2]}$ Only few cases of ovarian IMT have been reported. ${ }^{[3]}$

Exact etiology is unknown though, infection with inflammation has been proposed to be a causative factor. Recently the role of human herpes virus-8 DNA sequences and over expression of human interleukin 6 and cyclin D1 has been reported in seven cases. ${ }^{[6]}$ Cases of IMT have been reported post treatment of Wilm's tumour in oesophagus, stomach and liver. ${ }^{[7]}$ Recent report suggests that it is a neoplastic process because of its aggressive behaviour, involvement of chromosome 2p23 and cytogenetic clonality. ${ }^{[8]}$
The symptoms of IMT vary according to the site of involvement. Patients with intra-abdominal tumors most commonly complain of abdominal pain or an increasing abdominal mass and occasionally with signs and symptoms of gastrointestinal obstruction. Pulmonary IMT presents with chest pain and dyspnoea. ${ }^{[9]}$ Some patients have prominent systemic manifestations, including fever, night sweats, weight loss and malaise. Laboratory abnormalities are present in up to one - third of patients and include an elevated erythrocyte sedimentation rate, anemia, thrombocytosis, and polyclonal hypergammaglobulinemia, which often resolve when the lesion is excised. ${ }^{[10]}$ Computed tomography (CT) scan and 
magnetic resonance imaging (MRI) studies reveal a solid mass with infiltrative growth. ${ }^{[1]}$

Grossly, most lesions present as exophytic, nodular or polypoidal intraluminal lesions that may infiltrate deeply into visceral organs from which they arise and also into the adjacent structures. They range in size from $1.0 \mathrm{~cm}$ to upto $17 \mathrm{~cm} \cdot{ }^{[1]}$ The consistency of lesion depends upon the relative amounts of fibrous and myxoid stroma present.

Microscopically, IMT is composed of myofibroblasts and inflammatory cells. The spindled myofibroblasts and inflammatory cells of IMT are arranged in three basic histological patterns ${ }^{[5]}$ In first pattern, myofibroblasts are loosely arranged in an oedematous myxoid background with abundance of blood vessels and chronic inflammatory infiltrate comprising of plasma cells, lymphocytes and eosinophils. This pattern mimics granulation tissue, nodular fasciitis, or other reactive processes. A second pattern is characterised by fascicular and storiform arrangement of myofibroblasts with variable myxoid and collagenised areas and mature lymphoid and plasma cell infiltrate with germinal centres.. Ganglion like myofibroblast are often seen in these two patterns. This pattern resembles fibromatosis, fibrous histiocytoma, or smooth muscle neoplasm. The third pattern is paucicellular with sparse inflammatory infiltrate and dense collagenisation. It resembles a scar or desmoids-type fibromatosis. Highly atypical polygonal cells with vesicular nuclei, prominent nucleoli and variable mitoses are seen in rare IMTs which undergo histologic malignant transformation. ${ }^{[5,12]}$ Invasion into the muscularis propria of the urinary bladder is a common finding and some even infiltrate into the perivascular adipose tissue. IMT needs to be differentiated from IgG4 related disease due to the prominent plasma cell infiltrate and fibrosis in both the lesions. ${ }^{[1]}$ From an immunophenotypic standpoint, the spindle cells of IMT expresses diffuse cytoplasmic reactivity for vimentin. ${ }^{[1]}$ ALK positivity is detectable in approximately $50 \%$ of IMTs. ${ }^{[1,14]}$ Focal to diffuse reactivity for smooth muscle actin and muscle specific actin is also demonstrated in spindle cells cytoplasm. ${ }^{[1,14]}$ One third of cases show immunoreactivity for cytokeratin. Sometimes it is mistaken as immunoglobulin G4 subclass (IgG4) related sclerosing disease, however IMT is negative for IgG4. ${ }^{[1]}$

Surgery is the principal modality of treatment. Extrapulmonary IMTs have a recurrence rate of $25 \%$ and depends upon anatomical site, resectability and multinodularity. ${ }^{[1]}$ Metastasis is also reported in $<5 \%$ of cases. ${ }^{[1]}$ Factors related to adverse prognosis are morphological atypia, ganglion like cells, expression of TP53 and aneuploidy. ${ }^{[12]}$
The differential diagnosis of IMT comprises of low grade myofibroblastic sarcoma, benign reactive or neoplastic mesenchymal lesions such as, solitary fibrous tumour, spindle cell carcinoma, nodular fasciitis and peripheral nerve sheath tumour.

\section{Conclusion}

The clinicians and pathologists should be familiar with this entity and diagnosis of IMT should be considered in a mass presenting with inflammatory reactions. As IMT is a benign mimicker of malignancy, histopathological examination is mandatory for its diagnosis and treatment. Misdiagnosis can lead to unnecessary morbidity and mortality related to extensive debulking surgeries and even chemotherapy.

\section{Reference}

1. Fletcher CDM, Bridge JA, Hogendoorn PCW et al. Inflammatory myofibroblastic tumour. In: Fletcher CDM, Bridge JA, Hogendoorn PCW, Mertens F, editors. Lyon International Agency for Research on Cancer. World Health Organization Classification of Tumours of Soft Tissue and Bone. 4th edition;2013: 83-4.

2. Olgan, S, Saatli, B et al. Hysteroscopic excision of inflammatory myofibroblastic tumor of the uterus: as case report and brief review. Eur. J. Obstet. Gynecol Reprod. Biol.2011;157: 234-240.

3. Shintaku, M, Fukushima A. Inflammatory myofibroblastic tumor of the uterus with prominent myxoid change. Pathol. Int.2006; $56: 625-628$.

4. Bharatnur S, Ramkumar V, K Natrajan. Inflammatory Pseudo-Tumor Of Ovary - A Case Report. The Internet Journal of Gynecology and Obstetric.2012;16:1.

5. Sahnoun L, Elezzi O, Maazoun K, Krichene I et al. Ovarian inflammatory myofibroblastic tumor in children. J Pediatr Adolesc Gynecol. 2007 ;20(6):365-6.

6. Wang TY, Chou JW, Shih YS et al. Inflammatory myofibroblastic tumor mimicking adrenal incidentaloma. Intern Med. 2011;50: 165-6.

7. Coffin CM, Humphrey PA, Dehner LP . Extrapulmonary inflammatory myofibroblastic tumor: a clinical and pathological survey. Semin Diagn Pathol .1998;15: 85-101.

8. Coffin CM, Watterson J, Priest JR, Dehner LP. Extrapulmonary inflammatory myofibroblastic tumor (inflammatory pseudotumor). A clinicopathologic and immunohistochemical study of 84 cases. Am J Surg Pathol.1995; 19: 859-872.

9. Gomez-Roman JJ, Vinyals GO,Velasco PS et al. Presence of human herpesvirus- 8 DNA sequences and overexpression of human IL-6 and cyclin D1 in inflammatory myofibroblastic tumor (inflammatory pseudotumor). Lab Invest.2006; 80:1121-1126. 
10. Vujanic GM, Milovanovic D, Aleksandrovic S. Aggressive inflammatory pseudotumor of the abdomen 9 years after therapy for Wilms tumor. A complication, coincidence, or association? Cancer.1992; 70: 2362-2366.

11. Poh CF, Priddy RW, Dahlman DM. Intramandibular inflammatory myofibroblastic tumour: A true neoplasm or reactive lesion? Oral Surg Oral Med Oral Pathol Oral Radiol Endod. 2005;100:460-6.

12. Volker HU, Scheich M, Holler S et al. Differential diagnosis of laryngeal spindle cell carcinoma and inflammatory myofibroblastic tumour: Report of two cases with similar morphology. Diagn Pathol. 2007;2:1-7.

*Corresponding author:

DR.IFFAT JAMAL, Flat No-D/2,Phase-1,Sapna Apartment, Nayatola, Patna(Bihar)-800004 India

Phone: +91 9835498843

Email: iffatjamal111@gmail.com

Date of Submission : 15.01.2017

Date of Acceptance : 03.04.2017

Financial or other Competing Interests: None.

Date of Publication : 25.08.2017 